\title{
Global Sensitivity Analysis in Physiological Systems
}

\section{Tahmineh Azizi1, Robert Mugabi2}

${ }^{1}$ Institute of Computational Comparative Medicine, Department of Anatomy and Physiology, Department of Mathematics, Kansas State University, Manhattan, KS, USA

${ }^{2}$ Department of Diagnostic Medicine, and Pathobiology, College of Veterinary Medicine, Kansas State University, Manhattan, KS, USA

Email: tazizi@ksu.edu

How to cite this paper: Azizi, T. and Mugabi, R. (2020) Global Sensitivity Analysis in Physiological Systems. Applied Mathematics, 11, 119-136.

https://doi.org/10.4236/am.2020.113011

Received: January 30, 2020

Accepted: March 2, 2020

Published: March 5, 2020

Copyright $\odot 2020$ by author(s) and Scientific Research Publishing Inc. This work is licensed under the Creative Commons Attribution International License (CC BY 4.0).

http://creativecommons.org/licenses/by/4.0/

\begin{abstract}
Pharmacokinetic models are mathematical models which provide insights into the interaction of chemicals with biological processes. During recent decades, these models have become central of attention in industry that caused to do a lot of efforts to make them more accurate. Current work studies the process of drug and nanoparticle (NPs) distribution throughout the body which consists of a system of ordinary differential equations. We use a tri-compartmental model to study the perfusion of NPs in tissues and a six-compartmental model to study drug distribution in different body organs. We have performed global sensitivity analysis by LHS Monte Carlo method using PRCC. We identify the key parameters that contribute most significantly to the absorption and distribution of drugs and NPs in different organs in body.
\end{abstract}

\section{Keywords}

Global Sensitivity Analysis, Latin Hypercube Sampling (LHS), Partial Rank Correlation Coefficient (PRCC), Physiological Systems, Drug and NPs

Distribution

\section{Introduction}

Nanotechnology is the study of materials, devices, and systems at the nanometer scale. Nanotechnology and nanoscience have been used widely in many areas of research and applications [1] [2] [3] [4] [5]. One of the most important advantages of nanotechnology is that the drug can be targeted to a precise location which would make the drug much more effective and it also reduces the possible 
side effects. The application of nanotechnology in the field of nanomedicine and health care has grown a huge attention in recent times. Nanomedicine is a branch of nanotechnology. Basically, we can define nanomedicine as the medical application of nanotechnology [1] [2] [6]. Nanomedicine has many advantages over conventional drug delivery approaches and has been used frequently in anticancer research. There are some techniques which help in the detection of tumors in the body and nanoparticles are one of them [6]. Nanoparticles (NPs) help us to see cells and molecules that cannot be otherwise detected through conventional imaging. The abilities to understand what happens inside the cell and to observe therapeutic intervention and or to see when a cancer cell is lethally injured or is stimulated are important to the effective diagnosis and then better treatment of the disease [6] [7]. Nanoparticles (NPs) have unique physicochemical properties, such as small size, large surface area to mass ratio, and high reactivity, which are different from bulk materials of the same composition [1]. Indeed, because of these unique properties, NPs have very important role in anticancer therapy [2].

Mathematical and statistical modeling helps us to understand the interaction between the components of systems biology and prediction of the future of different biological models [8] [9] [10] [11] [12]. Basically, building a mathematical and computational model needs to perform different experiments and obtain different data which depicts the evolution of system [3] [4]. To understand the process of drug distribution through different body organs, we need to develop a comprehensive model which covers completely the experimental data [13] [14] [15]. These models transform all the information into a system of ordinary differential equations to do more analysis based on some mathematical useful tools and are flexible to analysis, updates and modifications of pharmacology and physiology of agents and drugs. A mathematical model for drug or NP distributions is a structural model, consisting of compartments such as adipose, tissues, brain, gut, heart, kidney, liver, lung, muscle, spleen, skin, and bone and gastrointestinal tract including mouth, esophagus, and abdomen which are connected by the cardiovascular system. In mathematical perspective, they describe biological systems by converting into mathematical and theoretical equations and parameters and then using computer code to solve the model system computationally.

To check the accuracy of any mathematical model, we need to use different methods and because of existence of uncertainty in experimental data, it can be often complicated. Uncertainty and sensitivity analysis are useful techniques which help us to identify these uncertainties in data and then control them [9] [10] [16] [17]. Sensitivity analysis allows us to identify the parameter or set of parameters that have the greatest impact on the model output. It then provides useful information about which parameter or input makes the most variability in the model output. Generally, local sensitivity analysis allows us to clarify the impact of each parameter on model outputs individually. However, global sensi- 
tivity analysis overcomes the limitations which the local sensitivity analysis creates by examining the sensitivity of model output over the entire range of parameters at the same time.

Current work studies the process of drug distribution throughout the body which consists of a system of ordinary differential equations. There are several biological parameters related to distribution of drug through different body organs. We start with a simple three compartmental model to demonstrate NP distribution from capillary to tissue. Globally sensitivity analysis LHS Monte Carlo method using Partial Rank Correlation Coefficient (PRCC) has been performed to investigate the key parameters in model equations. Also, we study a six compartmental system for which we assume the specific drug has been distributed through different rout of drug administrations, such as intravenous injection, intramuscular injection, water and or feed. We have used the same global sensitivity analysis PRCC method to compare different physiological parameters. We have used the parameters variations based on different studies [4] [18] [19] [20].

\section{A Simple Three Compartmental Model Example for NP Distribution}

There are some efforts to develop physiologically based pharmacokinetic models for nanoparticles distribution through the body, which will be useful tools for predicting nanoparticle distribution in different organs to assist with extrapolation of responses from in vitro and in vivo [21]. However, since the blood-flow limited model which has been used for chemicals [22] cannot be used for nanoparticles, we need to explain the distribution of the nanoparticles in the tissues to develop a model [21] [23] [24]. There are many studies about the effects of Nanomaterials on biological procedures like isolated in vitro cell system and or in vivo effects. But, there are only a few studies about vascular effects of Nanomaterials of different compositions [4] [25] [26] [27]. In some studies, researchers have considered Endothelial cells (EC) as in vitro model system for different physiological processes [28] [29] [30] [31]. According to these works, primary EC have a limited life span and demonstrate distinct characteristics that are different from each other. All vessels in body are covered with a line of Endothelial cells and these cells have different rolls based on their size and location in blood vessels. Some of their important tasks in our body are transporting small molecules and hormones like insulin, and degrading lipoprotein particles. Moreover, they affect blood pressure regulation and transport inflammatory cells into target tissue. Also, they have key role in blood coagulation and fibrinolysis. Endothelial cells are like barriers between blood cells and tissue cells [27]. They may help to some disorders like bleeding disorders, autoimmune disorders, or in pathological processes.

As we can see in Figure 1, EC or endothelial cells have covered blood vessels and they transport NPs from capillary to target tissue. We have used a tri compartmental model for NPs infusion to tissue. 


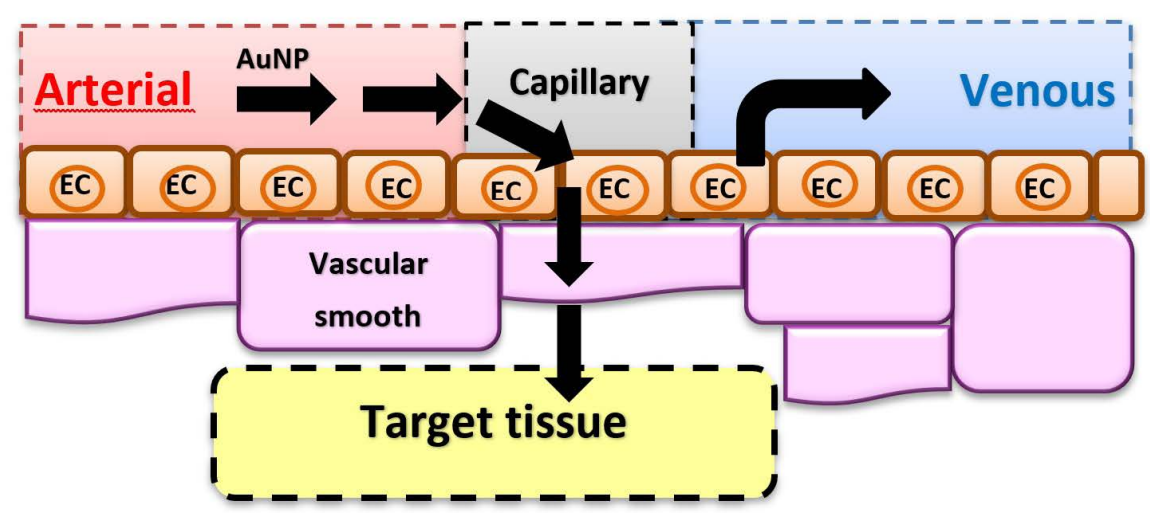

Figure 1. Endothelial cells as barriers between blood vessels and target tissue.

For NPs that move from blood vessels into different tissues this tricompartmental model is needed to characterize NPs infusion in the body. For simplicity, we have supposed that there is no interaction between Endothelial cells and their surrounding cells.

As we can see in Figure 2, NPs enter from artery to the second compartment by a constant rate $k_{12}$, we call this rate as absorption rate constant to capillary bed. After this step, NPs distribute to the third compartment by a constant rate $k_{23}$. Then, we have distribution of NPs into fixed or deep tissue compartment by the constant rate $k_{34}$. So far, we have finished two phases, absorption and distribution, and the last phase would be NPs leakage or NPs elimination.

If we apply the mass balance laws to this tri-compartmental model, we have:

$$
\left\{\begin{array}{l}
\frac{\mathrm{d} A_{\text {Cap }}}{\mathrm{d} t}=k_{12} A_{\text {Art }}-k_{23} A_{\text {Cap }}-k_{25} A_{\text {Cap }}+k_{32} A_{E C} \\
\frac{\mathrm{d} A_{E C}}{\mathrm{~d} t}=k_{23} A_{C a p}-k_{34} A_{E C}-k_{32} A_{E C} \\
\frac{\mathrm{d} A_{D T}}{\mathrm{~d} t}=k_{34} A_{E C}
\end{array}\right.
$$

We have two possible routes for NPs in compartment two. First possibility, they can distribute to compartment 3 and or they leave compartment two via venous efflux. Here, also we have assumed that the uptake depends on NP concentration in compartment two and it does not depend on perforate blood flow [4] [32]. For the first compartment, we can easily obtain the following equality:

$$
J_{25}=J_{12}-k_{23} A_{\text {Cap }}+k_{32} A_{E C}
$$

where $J_{25}=k_{25} A_{\text {Cap }}$ and $J_{12}=Q C_{12}$ ( $k_{25}$ is a variable rate function).

Physiologically speaking, we have considered that at starting time $t=0$ NPs enter from artery to compartment 2 and then they leave capillary bed to shallow tissue. Before the time for the venous effluent, venous efflux of NPs is zero, and at this moment, $t=\tau$, and then after that the sum of NPs fluxes to shallow tissue compartment and venous effluent should be equal to arterial flux or $J_{12}$. By this assumption that $\tau$ is small at steady state, we can compute the initial mass $M_{2}$ for capillary bed compartment that would be $M_{2}=C_{12} V_{2}$. Here, $C_{12}$ is 


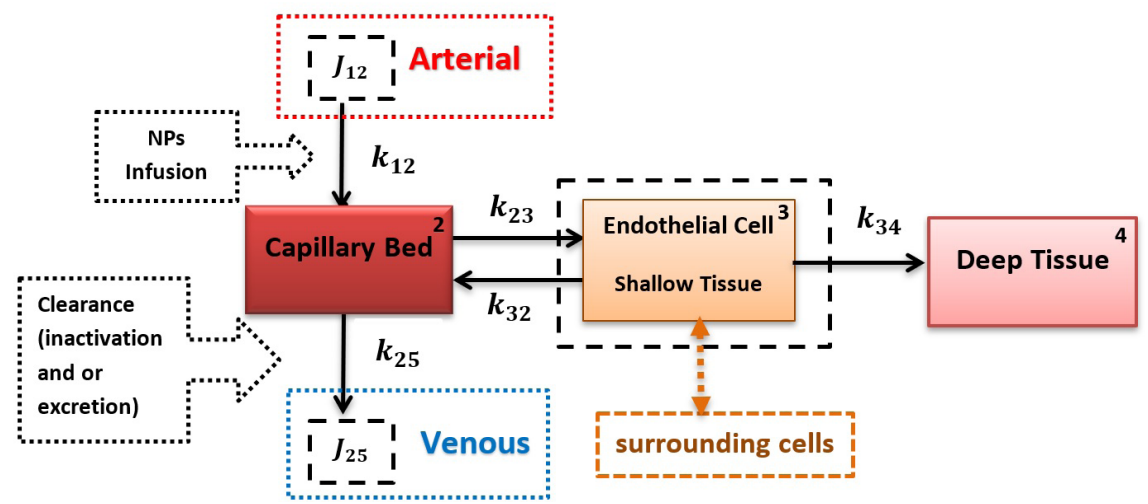

Figure 2. Structure of the tri-compartment pharmacokinetic model for nanoparticle disposition with considering NPs infusion, where $k_{23}, k_{32}$ and $k_{34}$ are transfer rate constants, $k_{25}$ describes the rate of mass transfer from vascular (2) compartment to the venous effluent, $J_{12}$ is the infusion drug flux, which is the product of flow $(Q)$ and concentration $\left(C_{12}\right)$.

the concentration of infused NPs, and $V_{2}$ is the vascular volume. Also, we can calculate the capillary transit time by the following equality $\tau=V_{2} / Q$, where, $Q$ is perfusate flow through skin flap and we consider it as a constant approximately equal $1 \mathrm{~mL} / \mathrm{min}$. The value of $k_{25}$ as a variable rate function after the time that flux $k_{32} A_{E C}$ reaches to compartment two increased and we will prove it by some computations later [4]. After solving model (2.1) for $A_{C a p}$ and $A_{E C}$ we have,

$$
\begin{gathered}
A_{C a p}=M_{2} \\
A_{E C}=\frac{M_{2} k_{23}}{k_{32}+k_{34}}\left(1-\mathrm{e}^{-\left(k_{32}+k_{34}\right) t}\right)+A_{E C}(0) \mathrm{e}^{-\left(k_{32}+k_{34}\right)(t-\Gamma)} \\
A_{D T}=\frac{M_{2} k_{23} k_{34}}{k_{32}+k_{34}}\left(t-\frac{1-\mathrm{e}^{-\left(k_{32}+k_{34}\right) t}}{k_{32}+k_{34}}\right)+\frac{k_{34} A_{E C}(0)}{k_{32}+k_{34}}\left[1-\mathrm{e}^{-\left(k_{32}+k_{34}\right) t(t-\Gamma)}\right]+A_{D T}(0)
\end{gathered}
$$

where we consider $\Gamma$ as the beginning time of washout phase that is zero during dosing phase [32]. Also, $A_{E C}(0)$ is the initial mass of compartment three and $A_{D T}(0)$ is the initial mass of compartment four. During dosing phase, $A_{E C}(0)=0$ and $A_{D T}(0)=0$. During washout phase, the initial mass of NPs in all compartments and $\Gamma$ is non-zero. In decay or washout phase, we can calculate $k_{25}$ at $t=0$ by the following equation [32]:

$$
k_{25}^{0}=\frac{J_{12}+k_{32} A_{E C}(0)}{A_{\text {Cap }}(0)}-k_{23}
$$

Because at $t=0, A_{E C}(0)=0$ and $A_{C a p}(0)=M_{2}$, so we have:

$$
k_{25}^{0}=\frac{J_{12}}{M_{2}}-k_{23}
$$

Therefore, $k_{25}$ at steady-state has the following value:

$$
k_{25}^{s s}=\frac{J_{12}+k_{32} A_{E C}(s s)}{A_{\text {Cap }}(s s)}-k_{23}
$$


such that $A_{\text {Cap }}^{\text {ss }}=M_{2}$ and

$$
A_{E C}^{s S}=\lim _{t \rightarrow \infty} A_{E C}(t)=\frac{k_{23} M_{2}}{k_{32}+k_{34}}
$$

If we substitute the value of $A_{C a p}^{s s}$ and $A_{E C}^{s s}$ into $k_{25}^{s s}$, then we have:

$$
k_{25}^{s s}=\frac{\left(k_{32}+k_{34}\right) J_{12}+k_{32} k_{23} M_{2}}{\left(k_{32}+k_{34}\right) M_{2}}-k_{23}
$$

and because we have, $\frac{J_{12}}{M_{2}}=\tau^{-1}$, so;

$$
k_{25}^{s s}=\tau^{-1}+\frac{k_{32} k_{23}}{k_{32}+k_{34}}-k_{23}
$$

and when $k_{34}=0$, we have $k_{25}^{s s}=\tau^{-1}$. Moreover, we can write $k_{25}^{0}=\tau^{-1}-k_{23}$. When we compare $k_{25}^{0}$ and $k_{25}^{\text {ss }}$, we see that $k_{25}^{0}$ is less than $k_{25}^{\text {ss }}$ by the following result:

$$
k_{25}^{s s}=k_{25}^{0}+\frac{k_{32} k_{23}}{k_{32}+k_{34}}
$$

During dosing phase $A_{\text {Cap }}=0$. At $t=\Gamma, A_{\text {Cap }}$ changes from $M_{2}$ to 0 , and also $A_{E C}(0)$ from 0 to $A_{E C}(\Gamma)$. During decay phase $J_{12}$ is non-zero. Figure 3 displays the evolution of the solutions of (2.1) in time and also with respect to other solutions in separate plots.
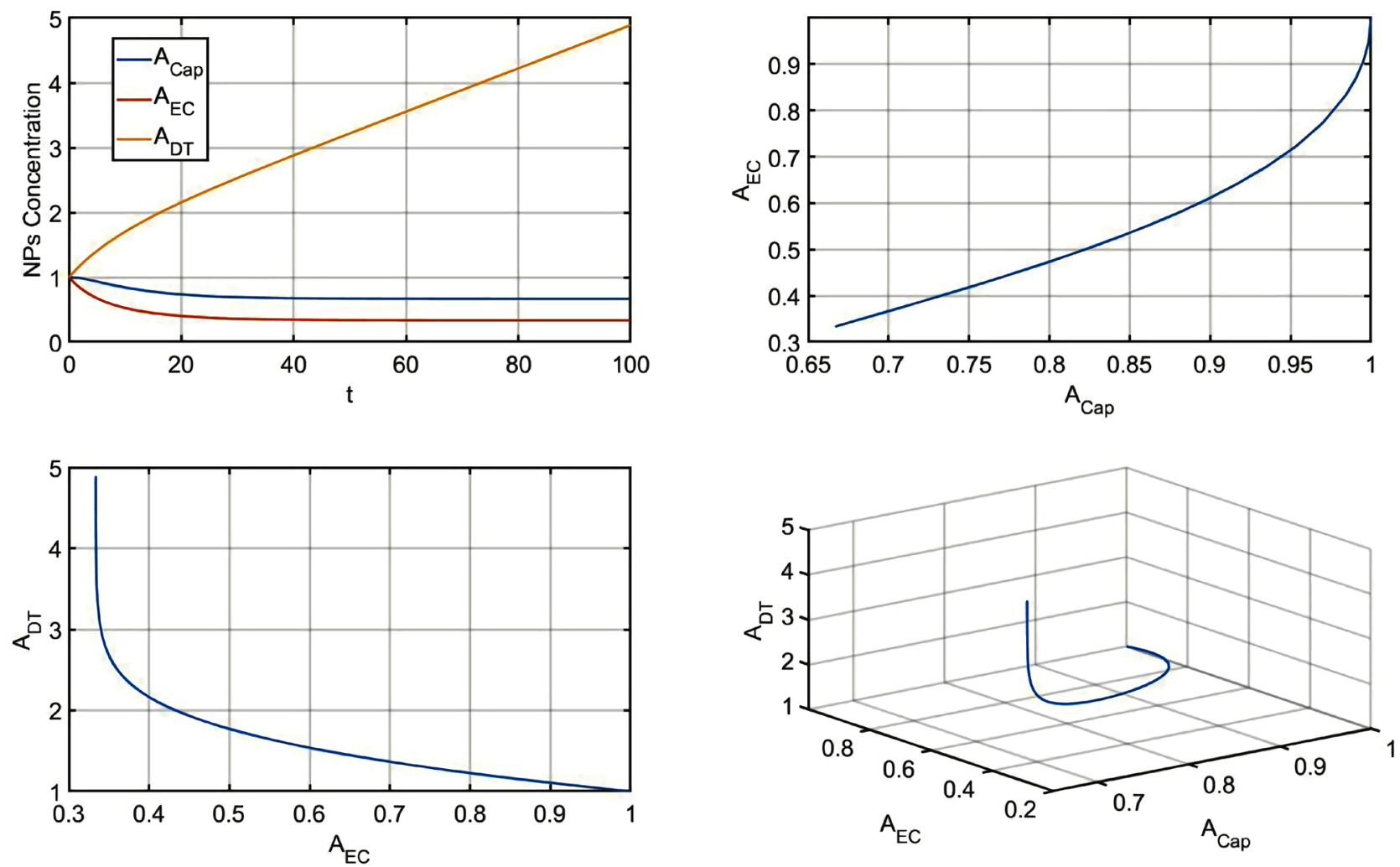

Figure 3. $A_{\text {Cap }}, A_{E C}$ and $A_{D T}$ in model (2.1). 


\section{A Six Compartmental Model Example for Drug Distribution through Body Organs}

Physiological and pharmacokinetic models are useful to determine drug distribution into different target tissues, which helps for the evaluation of drug efficacy and drug safety. We study a six-compartmental pharmacokinetic model with application in food safety and we use the physiological parameters variations based on different studies [18] [19].

Cardiac output and blood flows to tissues $(\mathrm{L} / \mathrm{h})$ :

$$
\left\{\begin{array}{l}
Q C=Q C C \times B W, \quad \text { Cardiacoutput } \\
Q L=Q L C \times Q C, \quad \text { Liver } \\
Q K=Q K C \times Q C, \quad \text { Kidney } \\
Q L u=Q L u C \times Q C, \quad \text { Lung } \\
Q F=Q F C \times Q C, \quad \text { Fat } \\
Q M=Q M C \times Q C, \quad \text { Muscle } \\
Q R=Q R C \times Q C, \quad \text { Restofbody }
\end{array}\right.
$$

where, $Q C C=4.944$ is cardiac output $(\mathrm{L} / \mathrm{h} / \mathrm{kg}), Q L C=0.2725$ is fraction of blood flow to the liver (unitless), $Q K C=0.12$ is fraction of blood flow to the kidneys (unitless), $Q F C=0.1275$ fraction of blood flow to the fat (unitless), $Q M C=0.251$ is fraction of blood flow to the muscle (unitless), $Q L u C=1$ is fraction of blood flow to the Lung (unitless), $Q R C=1-Q L C-Q K C-Q F C-Q M C$; $-Q L u C$ is fraction of blood flow to the rest of body and $B W$ is body weight [18].

Tissue volumes (L):

$$
\left\{\begin{array}{l}
\text { Vven }=\text { Vven } C \times B W, \\
\text { Vart }=\operatorname{Vart} C \times B W, \\
V L=V L C \times B W, \quad \text { Liver } \\
V K=V K C \times B W, \quad \text { Kidney } \\
V L u=V L u C \times B W, \quad \text { Lung } \\
V F=V F C \times B W, \quad \text { Fat } \\
V M=V M C \times B W, \quad \text { Muscle } \\
V R B=V R C \times B W, \quad \text { Restofbody }
\end{array}\right.
$$

where, $V L C=0.0245$ Fractional liver tissue (unitless), $V K C=0.004$ fractional kidney tissue, $V F C=0.32$ fractional fat tissue (unitless), $V M C=0.4$ fractional muscle tissue (unitless), $V L u C=0.010$ fractional Lung tissue (unitless), VvenC $=0.044$ venous blood volume, fraction of blood volume (unitless), VartC $=0.016$ Arterial blood volume, fraction of blood volume (unitless) and $V R C=1-V L C-V K C-V F C-V M C-V L u C-V v e n C-$ VartC fractional rest of body tissue (unitless).

Permeability surface area coefficients:

$$
\left\{\begin{array}{l}
P A F=P A F C \times V F, \\
P A M=P A M C \times V M,
\end{array}\right.
$$


where, permeability constants $(\mathrm{L} / \mathrm{h} / \mathrm{kg}$ tissue) (Permeation area cross products) are: $P A F C=0.012$ fat tissue permeability constant, $P A M C=0.225$ muscle tissue permeability constant [18].

Volume of tissue as blood:

$$
\left\{\begin{array}{l}
V F b=F V B F \times V F, \quad \text { Fat compartment blood volume } \\
V F t=V F-V F b, \quad \text { Fat compartment tissue volume }
\end{array}\right.
$$

where, $F V B F=0.02$ blood volume fraction of fat [20].

Muscle:

$$
\left\{\begin{array}{l}
V M b=F V B M \times V M, \quad \text { Muscle compartment blood volume } \\
V M t=V M-V M b, \quad \text { Muscle compartment tissue volume }
\end{array}\right.
$$

where, $F V B M=0.01$ blood volume fraction of muscle [20].

Dosing:

$$
\left\{\begin{array}{l}
\text { DOSEoral }=\text { PDOSEoral } \times B W,(\mathrm{mg}) \text { Oral dose } \\
\text { DOSEiv }=\text { PDOSEiv } \times B W,(\mathrm{mg}) \text { IV dose } \\
\text { DOSEim }=\text { PDOSEim } \times B W,(\mathrm{mg}) \quad \text { IM dose } \\
\text { DOSEoralw }=\text { PDOSEoralw } \times B W,(\mathrm{mg}) \text { Oral through water dose } \\
\text { DOSEoralf }=\text { PDOSEoralf } \times B W,(\mathrm{mg}) \quad \text { Oral through feed dose }
\end{array}\right.
$$

where, PDOSEoral , PDOSEiv, PDOSEim, PDOSEoralw and PDOSEoralf are parameters for exposure scenario.

Intramuscular (IM) injection equations:

$$
\left\{\begin{array}{l}
\operatorname{Rim}=\text { Kim } \times \text { Amtsite } \\
\frac{\mathrm{d}}{\mathrm{d} t}(\text { Absorb })=\text { Rim } \\
\text { Rsite }=- \text { Rim }+ \text { Kdiss } \times \text { Doseimremain } \\
\frac{\mathrm{d}}{\mathrm{d} t}(\text { Amtsite })=\text { Rsite } \\
\text { Rdoseimremain }=- \text { Kdiss } \times \text { Doseimremain } \\
\frac{\mathrm{d}}{\mathrm{d} t}(\text { Doseimremain })=\text { Rdoseimremain }
\end{array}\right.
$$

where, $\operatorname{Kim}=0.15$ or $\operatorname{Kim}=0.3 \quad I M \quad$ IM absorption rate constant $(/ \mathrm{h})$, Kdiss $=0.02$ IM absorption rate constant [18].

Intravascular (IV) injection to the venous equations:

$$
\left\{\begin{array}{l}
\text { IVR }=\text { DOSEiv } / \text { Timeiv } \\
\text { Riv }=I V R \times(1-\text { heaviside }(T-\text { Timeiv })) \\
\frac{\mathrm{d}}{\mathrm{d} t}(\text { Aiv })=\text { Riv }
\end{array}\right.
$$

where, Timeiv is IV injection/infusion time (h).

Urinary elimination rate constant:

$$
\text { Kurine }=\text { Kurine } C \times B W
$$

Liver compartment: 


$$
\left\{\begin{array}{l}
R L=Q L \times(C A-C V L)+R A O, \\
\frac{\mathrm{d}}{\mathrm{d} t}(A L)=R L, \\
C L=A L / V L, \\
C V L=A L /(V L \times P L), \\
\frac{\mathrm{d}}{\mathrm{d} t}(A U C C L)=C L,
\end{array}\right.
$$

Blood compartment:

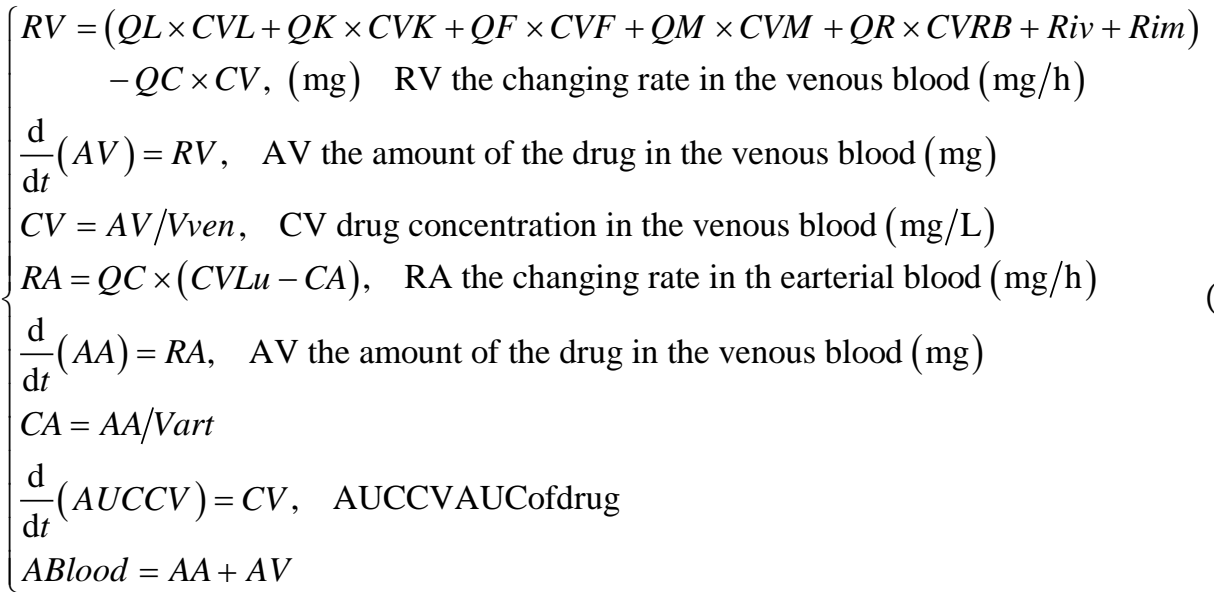

Kidney compartment:

$$
\left\{\begin{array}{l}
R K=Q K \times(C A-C V K)-\text { Rurine, } \\
\frac{\mathrm{d}}{\mathrm{d} t}(A K)=R K, \\
C K=A K / V K, \\
C V K=A K /(V K \times P K), \\
\frac{\mathrm{d}}{\mathrm{d} t}(A U C C K)=C K, \\
\text { Rurine }=\text { Kurine } \times C V K, \\
\frac{\mathrm{d}}{\mathrm{d} t}(\text { Aurine })=\text { Rurine },
\end{array}\right.
$$

Muscle compartment:

$$
\left\{\begin{array}{l}
R M B=Q M \times(C A-C V M)-P A M \times C V M+P A M \times C M t / P M, \\
\frac{\mathrm{d}}{\mathrm{d} t}(A M B)=R M B \\
C V M=A M B / V M B \\
R M t=P A M \times C V M-P A M \times C M t / P M, \\
\frac{\mathrm{d}}{\mathrm{d} t}(A M t)=R M t \\
C M t=A M t / V M t \\
A M \text { total }=A M t+A M B \\
C M=A M t o t a l / V M \\
\frac{\mathrm{d}}{\mathrm{d} t}(A U C C M)=C M
\end{array}\right.
$$


Lung compartment:

$$
\left\{\begin{array}{l}
R L u=Q L u \times(C V-C V L u), \\
\frac{\mathrm{d}}{\mathrm{d} t}(A L u)=R L u, \\
C L u=A L u / V L u, \\
C V L u=A L u /(V L u \times P L u), \\
\frac{\mathrm{d}}{\mathrm{d} t}(A U C C L U)=C L u,
\end{array}\right.
$$

Fat compartment:

$$
\left\{\begin{array}{l}
R F B=Q F \times(C A-C V F)-P A F \times C V F+P A F \times C F t / P F \\
\frac{\mathrm{d}}{\mathrm{d} t}(A F B)=R F B, \\
C V F=A F B / V F B, \\
R F t=P A F \times C V F-P A F \times C F t / P F, \\
\frac{\mathrm{d}}{\mathrm{d} t}(A F t)=R F t, \\
C F t=A F t / V F t, \\
\text { Aftotal }=A F t+A F B, \\
C F=\text { Aftotal } / V F,
\end{array}\right.
$$

Rest of body:

$$
\left\{\begin{array}{l}
R R B=Q R \times(C A-C V R B), \\
\frac{\mathrm{d}}{\mathrm{d} t}(A R)=R R B, \\
C R=A R / V R B, \\
C V R B=A R /(V R B \times P R), \\
\frac{\mathrm{d}}{\mathrm{d} t}(A U C C R)=C R,
\end{array}\right.
$$

Mass balance equation:

$\left\{\begin{array}{l}Q b a l=Q C-Q L-Q K-Q M-Q F-Q R, \\ \text { Tmass }=\text { Ablood }+A L+A K+\text { AMtotal }+ \text { AFtotal }+A R+\text { Aurine }+A L u, \\ \text { Bal = AAO }+ \text { Aiv }+ \text { Absorb }- \text { Tmass, Permeability-limited model mass balance }\end{array}\right.$

\section{Global Sensitivity Analysis}

Global sensitivity analysis allows us to change all parameters simultaneously over the entire parameter interval. This is a way to evaluate the relative effects of each input parameter and also to identify the interactions between parameters to the model output. In global sensitivity analysis we determine that with variation of input parameters in a certain range, which parameters and interactions have the most influential impact on the overall behavior of our model [8] [9] [10] [11] [16] [17].

There are several types of global sensitivity analyses, such as weighted average of local sensitivity analysis, partial rank correlation coefficient, multi parametric 
sensitivity analysis, Fourier amplitude sensitivity analysis (FAST) and Sobol's method, which can be used for systems pharmacology models [8]. The Latin hypercube sampling (LHS) method has been used frequently for global sensitivity analysis. There are also some other methods for calculating main effect and total effect sensitivity indices and one of the most important one is the method of Sobol [16].

LHS method is a sampling method and requires fewer samples compare to simple random sampling to achieve the same accuracy [8]. In LHS method, we divide the random parameter distributions into $N$ equal probability intervals. Here, $N$ is the sample size. The choice for $N$ should be at least $k+1$, where $k$ is the number of parameters which are varied. For the case that the interval of variation for some parameter is very large, the sampling can be done on a $\log$ form.

In LHS method, sampling is independent for each parameter and can be done by randomly selecting values from each $p d f$. We may sample each interval once for each parameter without any replacement. The LHS matrix is consisting of $N$ rows corresponding to the number of simulations or sample size and also it includes $k$ columns corresponding to the number of varied parameters. Then, $N$ model solutions may be simulated, using each combination of parameter values which they represent each row of the LHS matrix [8].

\subsection{Partial Rank Correlation Coefficient (PRCC) Results for Tri-Compartmental Model (2.1)}

Here, a parameter sensitivity analysis has being conducted to identify the pharmacokinetic parameters that have the most significant effect on our model system by the LHS Monte Carlo method using PRCC with uniform distributions for the 95 percent confidence intervals. The global sensitivity results with p-values corresponding to capillary compartment, endothelial cell compartment and deep tissue compartment have been demonstrated in Figures 4(1)-(3) respectively.

(1)
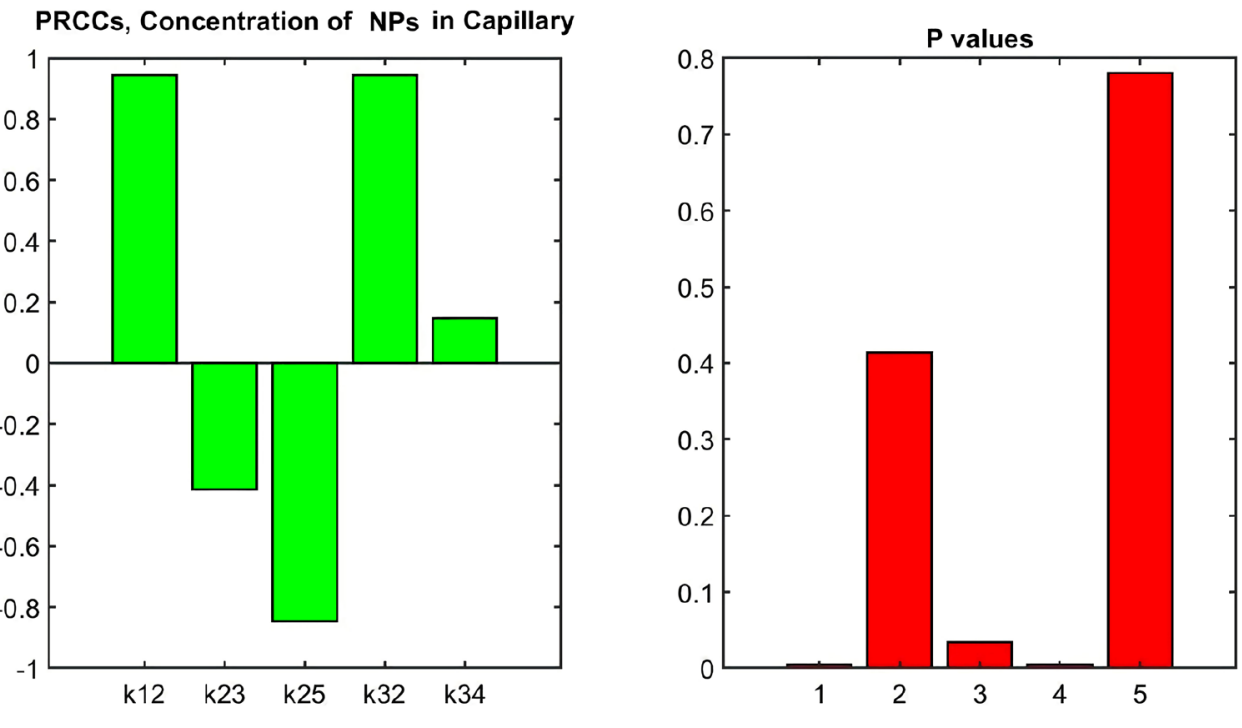
(2)

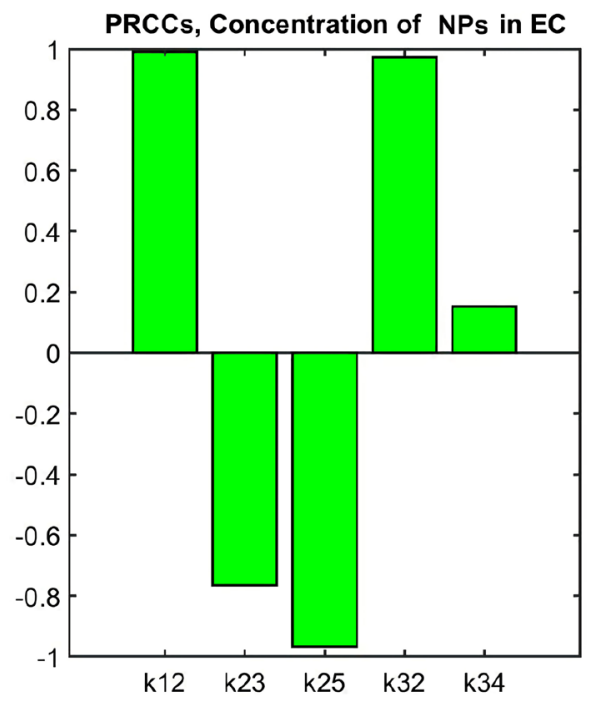

(3)

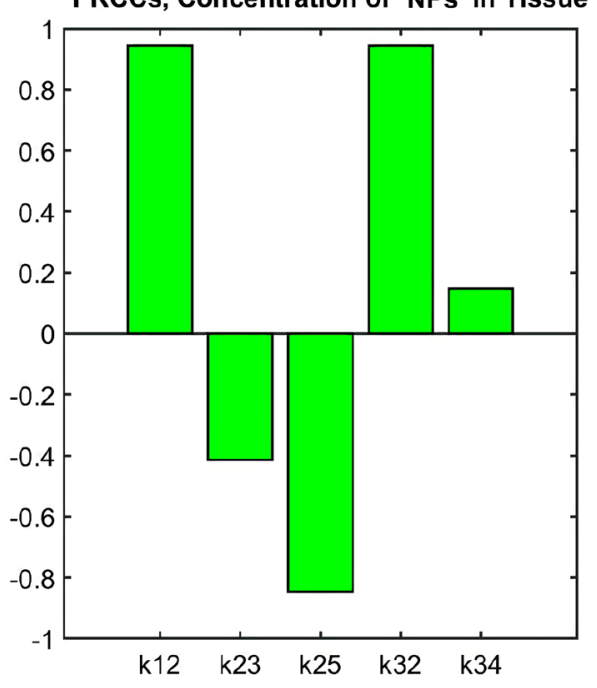

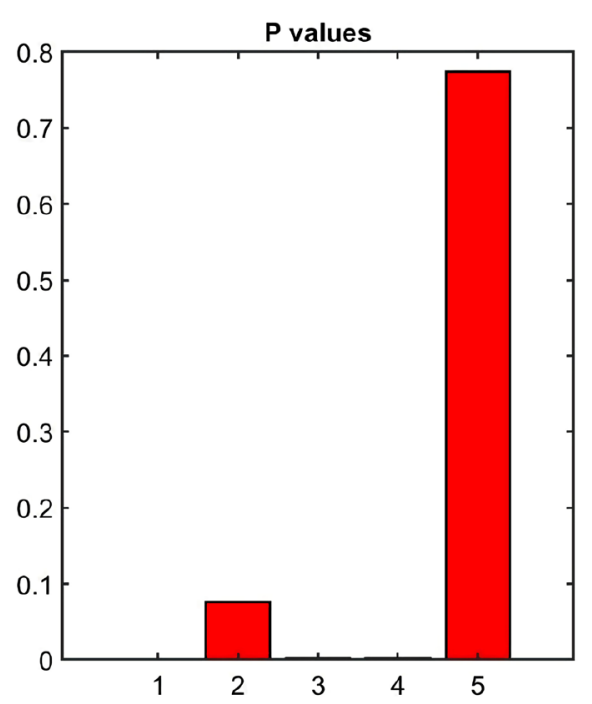

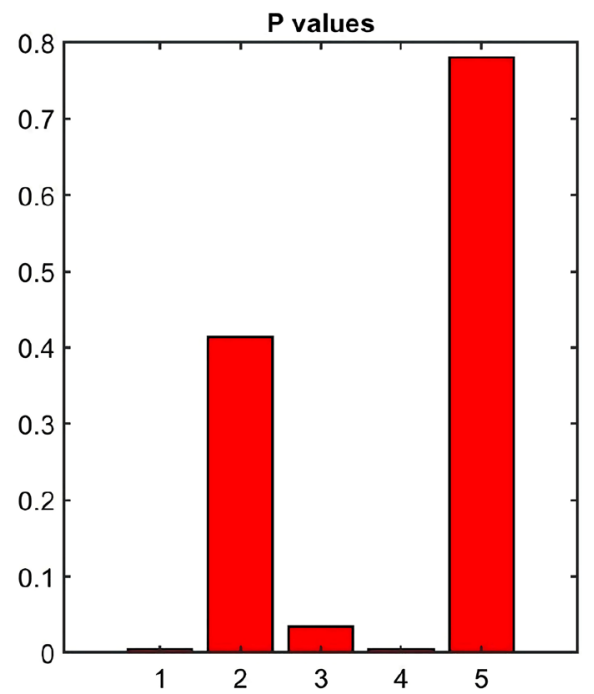

Figure 4. (1) Global uncertainty and sensitivity analysis of calculated different parameters for capillary compartment of model (2.1); (2) Global uncertainty and sensitivity analysis of calculated different parameters for endothelial cell compartment of model (2.1); (3) Global uncertainty and sensitivity analysis of calculated different parameters for deep tissue compartment of model (2.1).

\subsection{Partial Rank Correlation Coefficient (PRCC) Results for Six-Compartmental Model (3.11)-(3.14)}

According to LHS, we simulated the responses of the model for each organ by randomly selecting values for the parameter set from the 95 percent confidence intervals. These analyses were done by developing a LHS/ PRCC method with uniform distributions for the 95 percent confidence intervals. We found that some parameters illustrate significant performance in terms of sensitivity of the output to the variations of these parameters in some organs while they do not have this effect for other organs. These results have been depicted in Figure 5(A) for kidney tissue, Figure 5(B) for liver tissue, Figure 5(C) for lung tissue, Figure 5(D) for fat tissue, Figure 5(E) for muscle tissue and Figure 5(F) for plasma, are statistically significant with p-values much smaller than 0.01 . 


\section{Conclusions}

Nowadays, nanoparticles have a growing use in industry specially medicine. There are some studies about applications of NPs in therapeutic areas, however, the number of these studies is not a lot. Increasing the importance of studies about tumors and concentration of drugs and NPs in tumors or other tissues has enhanced the role of in vitro models to simulate absorption process of drugs and NPs. Pharmacokinetic and physiological models are useful means to demonstrate the relationships between different drug administrations, and drug exposure or concentration.
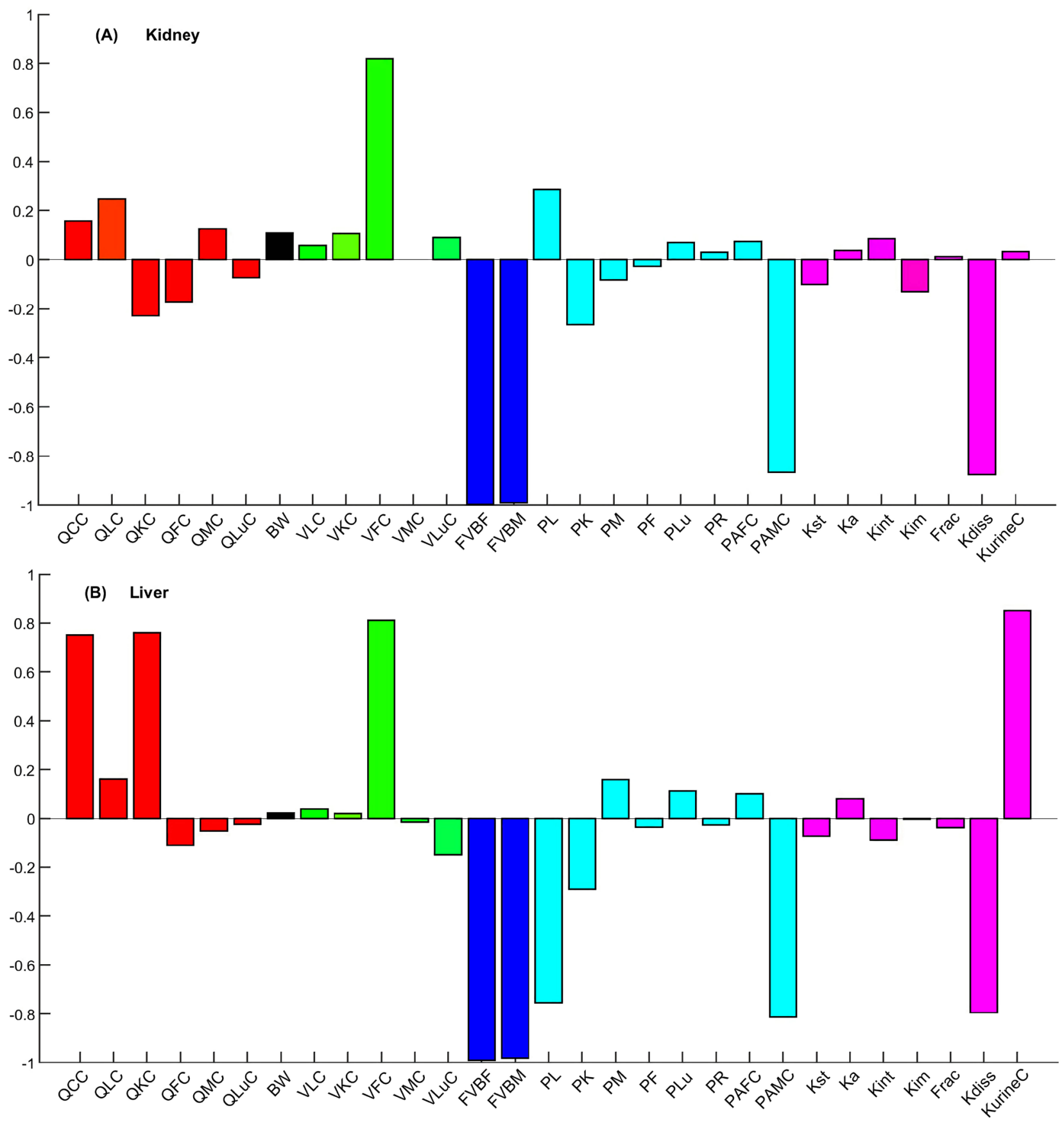

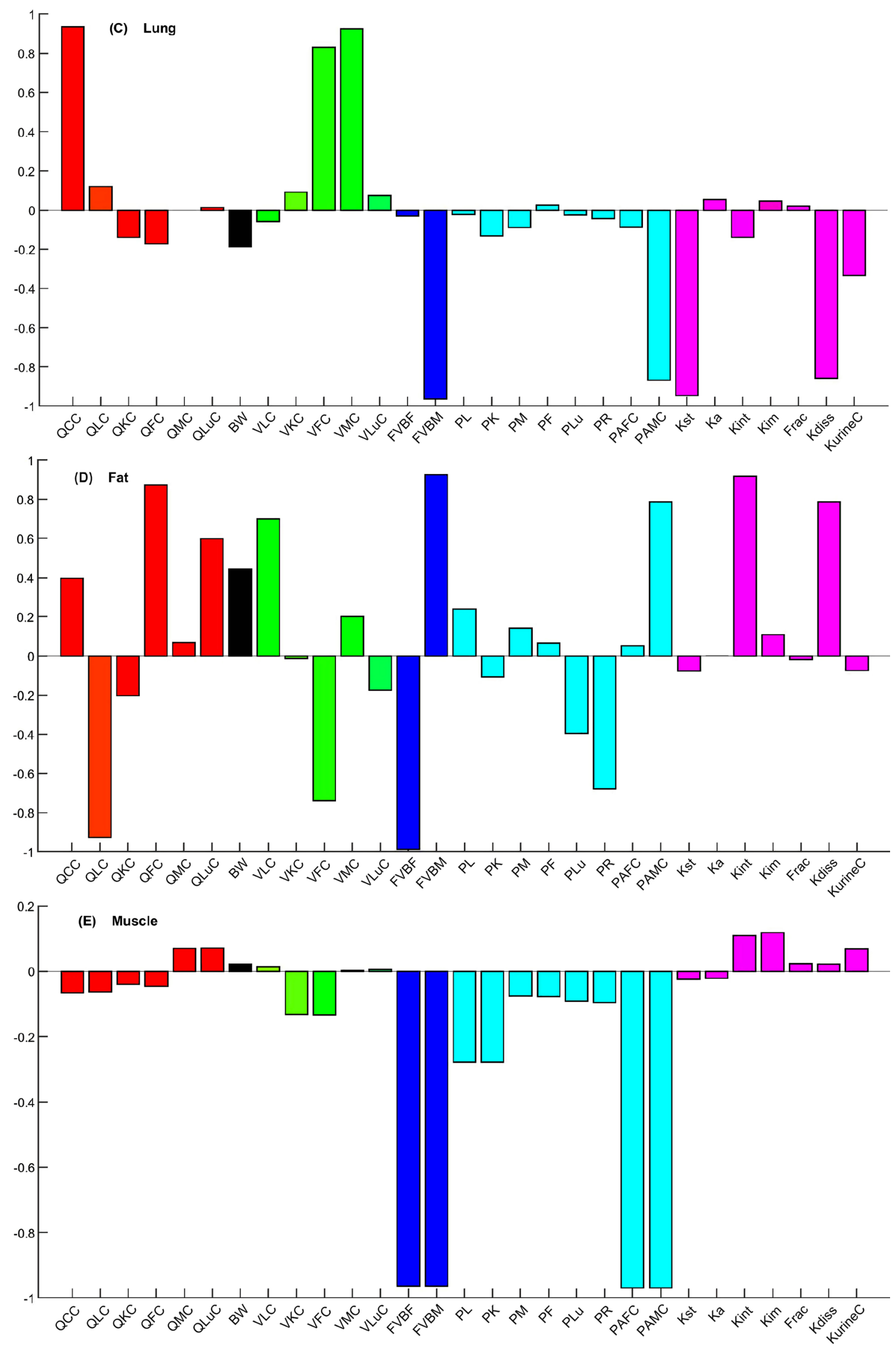


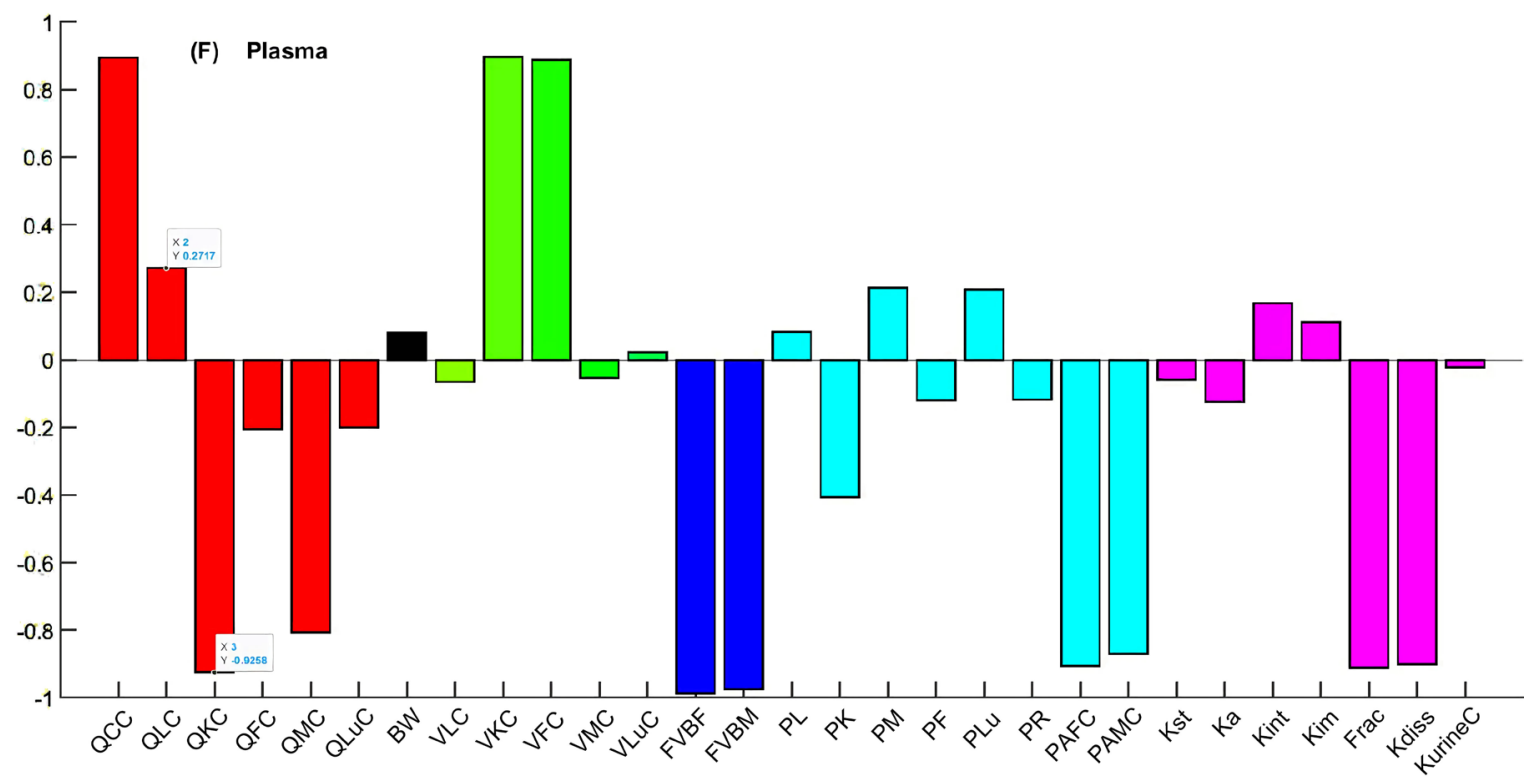

Figure 5. (A) Global uncertainty and sensitivity analysis of calculated different parameters for kidney. Analysis based on parameter effects for $C_{k}$ (concentration of drug in kidney). The PRCCs are compiled within the pharmacokinetic parameters ranges obtained from [18] [19] [20]; (B) Global uncertainty and sensitivity analysis of calculated different parameters for kidney. Analysis based on parameter effects for $C_{L}$ (concentration of drug in liver). The PRCCs are compiled within the pharmacokinetic parameters ranges obtained from [18] [19] [20]. (C) Global uncertainty and sensitivity analysis of calculated different parameters for kidney. Analysis based on parameter effects for $C_{L u}$ (concentration of drug in lung). The PRCCs are compiled within the pharmacokinetic parameters ranges obtained from [18] [19] [20]; (D) Global uncertainty and sensitivity analysis of calculated different parameters for kidney. Analysis based on parameter effects for $C_{F}$ (concentration of drug in fat). The PRCCs are compiled within the pharmacokinetic parameters ranges obtained from [18] [19] [20]; (E) Global uncertainty and sensitivity analysis of calculated different parameters for kidney. Analysis based on parameter effects for $C_{M}$ (concentration of drug in muscle). The PRCCs are compiled within the pharmacokinetic parameters ranges obtained from [18] [19] [20]; (F) Global uncertainty and sensitivity analysis of calculated different parameters for kidney. Analysis based on parameter effects for $C_{V}$ (concentration of drug in plasma). The PRCCs are compiled within the pharmacokinetic parameters ranges obtained from [18] [19] [20].

An uncertainty analysis may be applied on the physiological and pharmaceutics models to investigate the uncertainty in system output that is generated from uncertainty in parameter inputs. Sensitivity analysis assesses how variations in model outputs can be apportioned, qualitatively or quantitatively, to different inputs.

In this research we reviewed two physiological systems which have been reported by different authors and we have used the presented physiological parameters from different published works. In the first case, we presented a three compartmental model which can be used to exhibit the distribution of drug and or NPs from capillary compartment to endothelial cells compartment and then tissue compartment. The objective of this study was to determine the key parameters in NPs infusion from blood vessels to target tissue in the ex vivo tissue perfusion system using sampling-based method (Partial Rank Correlation Coefficient-PRCC). As we have seen, some parameters have positively and some others negatively affected NPs infusion process. 
We have presented another physiological model with six compartments, such as kidney, liver, lung, fat, muscle and plasma compartment. We identified the key parameters that contribute most significantly to the absorption and distribution of drugs in different organs in body using PRCC. Our findings imply that this identification is clearly dependent upon the dose and target tissues but not on the exposure route.

\section{Acknowledgements}

This work was supported by the Institute of Computational Comparative Medicine (ICCM) and department of Mathematics of Kansas State University. With a special thanks to Dr. Majid Jaberi-Douraki for his full support.

\section{Conflicts of Interest}

The authors declare no conflicts of interest regarding the publication of this paper.

\section{References}

[1] Earn, D.J.D., Rohani, P., Bolker, B.M. and Grenfell, B.T. (2012) Polymeric and Ceramic Nanoparticles in Biomedical Applications. Journal of Nanotechnology, 2012, Article ID: 936041. https://doi.org/10.1155/2012/936041

[2] Biswas, A.K., Islam, M.R., Choudhury, Z.S., Mostafa, A. and Kadir, M.F. (2014) Nanotechnology Based Approaches in Cancer Therapeutics. Advances in Natural Sciences. Nanoscience and Nanotechnology, 5, Article ID: 043001. https://doi.org/10.1088/2043-6262/5/4/043001

[3] Pitchaimani, A., Nguyen, T.D.T., Marasini, R., Eliyapura, A., Azizi, T., Jaberi-Douraki, M. and Aryal, S. (2019) Biomimetic Natural Killer Membrane Camouflaged Polymeric Nanoparticle for Targeted Bioimaging. Advanced Functional Materials, 29, Article ID: 1806817. https://doi.org/10.1002/adfm.201806817

[4] Riviere, J.E., Jaberi-Douraki, M., Lillich, J., Azizi, T., Joo, H., Choi, K., Thakkar, R. and Monteiro-Riviere, N.A. (2018) Modeling Gold Nanoparticle Biodistribution after Arterial Infusion into Perfused Tissue: Effects of Surface Coating, Size and Protein Corona. Nanotoxicology, 12, 1093-1112. https://doi.org/10.1080/17435390.2018.1476986

[5] Moreno-Vega, A.-I., Gomez-Quintero, T., Nunez-Anita, R.-E., Acosta-Torres, L.-S. and Castaño, V. (2015) Nanoparticles for Radiation Therapy Enhancement: The Key Parameters. Theranostics, 9, 1030.

[6] Shubhika, K. (2012) Nanotechnology and Medicine: The Upside and the Downside. The International Journal of Drug Development and Research, 5, 1-10.

[7] Mauricio, M.D., Guerra-Ojeda, S., Marchio, P., Valles, S.L., Aldasoro, M., Escribano-Lopez, I., Herance, J.R., Rocha, M., Vila, J.M. and Victor, V.M. (2018) Nanoparticles in Medicine: A Focus on Vascular Oxidative Stress. Oxidative Medicine and Cellular Longevity, 2018, Article ID: 6231482.

https://doi.org/10.1155/2018/6231482

[8] Marino, S., Hogue, I.B., Ray, C.J. and Kirschner, D.E. (2008) A Methodology for Performing Global Uncertainty and Sensitivity Analysis in Systems Biology. Journal of Theoretical Biology, 254, 178-196. https://doi.org/10.1016/j.jtbi.2008.04.011 
[9] Blower, S.M. and Dowlatabadi, H. (1994) Sensitivity and Uncertainty Analysis of Complex Models of Disease Transmission: an HIV Model, as an Example. International Statistical Review, 3, 229-243. https://doi.org/10.2307/1403510

[10] Zi, Z. (2011) Sensitivity Analysis Approaches Applied to Systems Biology Models. IET Systems Biology, 5, 336-346. https://doi.org/10.1049/iet-syb.2011.0015

[11] Dalberg, J., Gimenez, H., Keeley, A., Azizi, T., Xi, X. and Jaberi-Douraki, M. (2019) Local and Global Dynamics of Discrete Type 1 Diabetes Model.

[12] Azizi, T., et al. (2015) Dynamics of a Discrete-Time Plant-Herbivore Model. Caspian Journal of Mathematical Sciences, 4, 241-256.

[13] Zhao, P., Zhang, L., Grillo, J.A., Liu, Q., Bullock, J.M., Moon, Y.J., Song, P., Brar, S.S., Madabushi, R., Wu, T.C., et al. (2011) Applications of Physiologically Based Pharmacokinetic (PBPK) Modeling and Simulation during Regulatory Review. Clinical Pharmacology \& Therapeutics, 89, 259-267. https://doi.org/10.1038/clpt.2010.298

[14] Barrett, J.S., Della Casa Alberighi, O., Läer, S. and Meibohm, B. (2012) Physiologically Based Pharmacokinetic (PBPK) Modeling in Children. Clinical Pharmacology \& Therapeutics, 92, 40-49. https://doi.org/10.1038/clpt.2012.64

[15] Wagner, C., Zhao, P., Pan, Y., Hsu, V., Grillo, J., Huang, S.M. and Sinha, V. (2015) Application of Physiologically Based Pharmacokinetic (PBPK) Modeling to Support Dose Selection: Report of an FDA Public Workshop on PBPK. CPT: Pharmacometrics \& Systems Pharmacology, 4, 226-230. https://doi.org/10.1002/psp4.33

[16] Sobol, I.M. (1993) Sensitivity Estimates for Nonlinear Mathematical Models. Mathematical Modelling and Computational Experiments, 4, 407-414.

[17] Saltelli, A., Tarantola, S., Campolongo, F. and Ratto, M. (2004) Sensitivity Analysis in Practice: A Guide to Assessing Scientific Models. John Wiley \& Sons, Hoboken.

[18] Lin, Z., Gehring, R., Mochel, J.P., Lave, T. and Riviere, J.E. (2016) Mathematical Modeling and Simulation in Animal Health Part II: Principles, Methods, Applications, and Value of Physiologically Based Pharmacokinetic Modeling in Veterinary Medicine and Food Safety Assessment. Journal of Veterinary Pharmacology and Therapeutics, 39, 421-438. https://doi.org/10.1111/jvp.12311

[19] Lin, Z.M., Li, M.J., Gehring, R. and Riviere, J.E. (2015) Development and Application of a Multiroute Physiologically Based Pharmacokinetic Model for Oxytetracycline in Dogs and Humans. Journal of Pharmaceutical Sciences, 104, 233-243. https://doi.org/10.1002/jps.24244

[20] Brown, R.P., Delp, M.D., Lindstedt, S.L., Rhomberg, L.R. and Beliles, R.P. (1997) Physiological Parameter Values for Physiologically Based Pharmacokinetic Models. Toxicology and Industrial Health, 13, 407-484. https://doi.org/10.1177/074823379701300401

[21] Lankveld, D.P.K., Oomen, A.G., Krystek, P., Neigh, A., Troost de Jong, A., Noorlander, C.W., Van Eijkeren, J.C.H., Geertsma, R.E. and De Jong, W.H. (2010) The Kinetics of the Tissue Distribution of Silver Nanoparticles of Different Sizes. Biomaterials, 31, 8350-8361. https://doi.org/10.1016/j.biomaterials.2010.07.045

[22] Meek, M.E.B., Barton, H.A., Bessems, J.G., Lipscomb, J.C. and Krishnan, K. (2013) Case Study Illustrating the WHO IPCS Guidance on Characterization and Application of Physiologically Based Pharmacokinetic Models in Risk Assessment. Regulatory Toxicology and Pharmacology, 66, 116-129. https://doi.org/10.1016/j.yrtph.2013.03.005

[23] Lee, H.A., Leavens, T.L., Mason, S.E., Monteiro-Riviere, N.A. and Riviere, J.E. 
(2009) Comparison of Quantum Dot Biodistribution with a Blood-Flow-Limited Physiologically Based Pharmacokinetic Model. Nano Letters, 9, 794-799. https://doi.org/10.1021/nl803481q

[24] Lin, P., Chen, J.-W., Chang, L.W., Wu, J.-P., Redding, L., Chang, H., Yeh, T.-K., Yang, C.S., Tsai, M.-H., Wang, H.-J., et al. (2008) Computational and Ultrastructural Toxicology of a Nanoparticle, Quantum Dot 705, in Mice. Environmental Science \& Technology, 42, 6264-6270. https://doi.org/10.1021/es800254a

[25] Riviere, J.E., Bowman, K.F., Monteiro-Riviere, N.A., Dix, L.P. and Carver, M.P. (1986) The Isolated Perfused Porcine Skin Flap (IPPSF) I. A Novel in Vitro Model for Percutaneous Absorption and Cutaneous Toxicology Studies. Toxicological Sciences, 7, 444-453. https://doi.org/10.1093/toxsci/7.3.444

[26] Riviere, J.E., Leavens, T.L., Brooks, J.D. and Monteiro-Riviere, N.A. (2012) Acute Vascular Effects of Nanoparticle Infusion in Isolated Perfused Skin. Nanomedicine: Nanotechnology, Biology and Medicine, 8, 428-431. https://doi.org/10.1016/j.nano.2012.02.016

[27] Lee, H.A., Imran, M., Monteiro-Riviere, N.A., Colvin, V.L., Yu, W.W. and Riviere, J.E. (2007) Biodistribution of Quantum Dot Nanoparticles in Perfused Skin: Evidence of Coating Dependency and Periodicity in Arterial Extraction. Nano Letters, 7, 2865-2870. https://doi.org/10.1021/nl071563c

[28] Sacks, T., Moldow, Ch.F., Craddock, Ph.R., Bowers, T.K. and Jacob, H.S. (1978) Oxygen Radicals Mediate Endothelial Cell Damage by Complement-Stimulated Granulocytes. An in Vitro Model of Immune Vascular Damage. The Journal of Clinical Investigation, 61, 1161-1167. https://doi.org/10.1172/JCI109031

[29] Blackman, B.R., Garca-Carden, G. and Gimbrone Jr., M.A. (2002) A New in Vitro Model to Evaluate Differential Responses of Endothelial Cells to Simulated Arterial Shear Stress Waveforms. Journal of Biomechanical Engineering, 124, 397-407. https://doi.org/10.1115/1.1486468

[30] Gaillard, P.J., Voorwinden, L.H., Nielsen, J.L., Ivanov, A., Atsumi, R., Engman, H., Ringbom, C., de Boer, A.G. and Breimer, D.D. (2001) Establishment and Functional Characterization of an in Vitro Model of the Blood: Brain Barrier, Comprising a Co-Culture of Brain Capillary Endothelial Cells and Astrocytes. European Journal of Pharmaceutical Sciences, 12, 215-222. https://doi.org/10.1016/S0928-0987(00)00123-8

[31] Kumar, T.R.S. and Krishnan, L.K. (2001) Endothelial Cell Growth Factor (ECGF) Enmeshed with Fibrin Matrix Enhances Proliferation of EC in Vitro. Biomaterials, 22, 2769-2776. https://doi.org/10.1016/S0142-9612(01)00020-5

[32] Williams, P.L. and Riviere, J.E. (1989) Definition of a Physiologic Pharmacokinetic Model of Cutaneous Drug Distribution Using the Isolated Perfused Porcine Skin Flap. Journal of Pharmaceutical Sciences, 78, 550-555.

https://doi.org/10.1002/jps.2600780708 